\title{
El indice tobillo-brazo puede mejorar la predicción de eventos cardiovasculares
}

Ankle brachial index may improve cardiovascular risk prediction

Ankle Brachial Index Collaboration. JAMA 2008; 300: 197-208.

\section{Objetivo}

Determinar si el índice tobillo brazo de presión arterial* (ITBPA) medido con ecografía doppler manual (no con estetoscopio) provee información independiente sobre el riesgo de eventos cardiovasculares (CV) y muerte, respecto del puntaje de Framingham (PF) y si mejora su capacidad de predicción.

\section{Fuentes y selección de datos}

Estudios que hubieran incluido participantes de cualquier sexo y edad provenientes de la población general, sin antecedentes de enfermedad coronaria, a quienes se les hubiera medido el ITBPA con una técnica estandarizada y se los hubiera seguido para determinar su mortalidad total y CV.

Fueron identificados mediante una búsqueda en MEDLINE y en EMBASE y a través de información no publicada recabada entre expertos en el tema.

\section{Resultados}

El análisis fue realizado sobre la base de 16 estudios poblacionales prospectivos que incluyeron 24955 hombres y 23339 mujeres sin historia de enfermedad CV y con un seguimiento de 480.325 personas/año.
El riesgo de muerte $\mathrm{CV}$ a diez años en hombres con un ITBPA menor a 0,9 fue $18,7 \%$ (IC95\% 13,3 a 24,1$)$ mientras que con un ITBPA normal $(1,1$ a 1,4$)$ fue $4,4 \%(3,2$ a 5,7$)$ lo que implica un $\operatorname{RR} 4,2(3,3$ a 5,4$)$.

La mortalidad CV a diez años en mujeres con ITBPA menor a 0,9 fue $12,6 \%(6,2$ a 19$)$ y fue $4,1 \%(2,2$ a 6,1$)$ en quienes tenían un ITBPA normal (RR 3,5; IC95\% 2,4 a 5,1).

El riesgo de muerte global y CV permaneció elevado luego de ajustar por el PF. Para los hombres el RR fue 2,9 (2,3 a 3,7) y para las mujeres $3,0(2,0$ a 4,49$)$. Comparándolo con un ITBPA mayor a 0,9, uno menor a 0,9 se asoció con el doble de mortalidad total, mortalidad CV y eventos coronarios mayores, en cada uno de los estratos de riesgo del SF.

La inclusión del ITBPA en la estratificación de riesgo CV del PF determinaría una re-categorización del riesgo y por lo tanto una modificación en las recomendaciones terapéuticas actuales en aproximadamente el $19 \%$ de los hombres y en el $36 \%$ de las mujeres. Ver tabla 1.

Tabla 1: predicción del riesgo de eventos cardiovasculares a diez años de acuerdo a la regla de Framingham y el ajuste por el índice tobillo brazo de presión arterial (ITBPA).

\begin{tabular}{|c|c|c|c|c|c|c|}
\hline & & \multicolumn{5}{|c|}{ Riesgo de eventos cardiovaseulares a diez años y número de pacientes incluidos en cada estrato $(n)$} \\
\hline & & \multirow{2}{*}{$\begin{array}{l}\text { Sin ajustar por } \\
\text { el ITBPA }\end{array}$} & \multicolumn{4}{|c|}{ Ajustado por el índice tobillo brazo de presión arterial } \\
\hline & & & 0,9 & 0,91 a 1,1 & 1,11 a 1,4 & Mayor a 1,4 \\
\hline \multirow[t]{3}{*}{ Hombres } & Alto & $23 \%(8398)$ & $40 \%(1149)$ & $21 \%(3406)$ & $18 \%(3668)$ & $14 \%(175)$ \\
\hline & Intermedio & $13 \%(7392)$ & $16 \%(245)$ & $12 \%(2069)$ & $12 \%(4815)$ & $8 \%(263)$ \\
\hline & Bajo & $5 \%(5643)$ & $8 \%(76)$ & $5 \%(1076)$ & $4 \%(4255)$ & $5 \%(236)$ \\
\hline \multirow[t]{3}{*}{ Mujeres } & Alto & $27 \%(1418)$ & $44 \%(200)$ & $21 \% \quad(598)$ & $22 \%(577)$ & $34 \%(43)$ \\
\hline & Intermedio & $13 \%(5563)$ & $25 \%(558)$ & $12 \%(2429)$ & $11 \%(2433)$ & $13 \%(143)$ \\
\hline & Bajo & $11 \%(15505)$ & $21 \%$ & $10 \%(6192)$ & $9 \%(7909)$ & $11 \%(321)$ \\
\hline
\end{tabular}

En negrita se indican los subgrupos que cambian de categoría de riesgo de eventos cardiovasculares (bajo a intermedio, o intermedio a alto en las mujeres; y alto a intermedio, o intermedio a bajo en los varones) cuando se ajustan los resultados de la regla de predicción clínica de Framingham por el ITBPA. Al producirse este cambio de categorización (en las mujeres se incrementa el riesgo predicho, mientras que en los hombres desciende) también cambiará la intensidad de la intervención de reducción de riesgo cardiovascular a la que van a ser sometidos.

\section{Conclusiones}

El ITBPA mejoraría la precisión de la estimación del riesgo CV hecha a través de PF.
Palabras claves: riesgo cardiovascular, índice tobillo brazo, arteriopatía periférica. Key words: cardiovascular risk, ankle brachial index, peripheral arterial disease. Fuente de financiamiento: Sanofi-Aventis/BMS.

\footnotetext{
* El índice tobillo brazo de presión arterial se obtiene dividiendo la presión arterial sistólica obtenida en el tobillo con la obtenida en el brazo. Un valor menor a 0,9 tiene alta especificidad (98\%) y sensibilidad (82\%) para el diagnóstico de enfermedad arterial periférica, valores entre 0,9 y 1,1 se consideran en el límite, y valores mayores a 1,1 normales.
} 


\section{Comentario}

La presencia de enfermedad vascular periférica (EVP) aislada determina un riesgo de muerte CV $3 \%$ al año, similar al de los pacientes con angina crónica estable. Si a la EVP le sumamos la presencia de enfermedad coronaria y a esta, la de enfermedad cerebro-vascular el riesgo aumenta a 6 y $9 \%$ respectivamente. Por lo tanto, no existen dudas de la importancia de diagnosticar y tratar a los pacientes con EVP. Sin embargo, sabemos que la gran mayoría de los eventos coronarios se presentan en pacientes de bajo y moderado riesgo, por lo que es fundamental intentar predecir cuales de estos pacientes sufrirán un evento ${ }^{1,2}$.

Al aplicar un puntaje pronóstico se busca discriminar entre quienes sufrirán un evento y quienes no. EI PF es la regla de predicción clínica más difundida en la práctica. Sin embargo sabemos que presenta limitaciones y que su poder de discriminación es pobre en ciertas poblaciones ( $p$. ej. jóvenes y mujeres). Al analizar las curvas $\mathrm{ROC}^{\star}$ del presente trabajo vemos que la capacidad de discriminación del PF en hombres fue 0,646 -lo que quiere decir que el $64,6 \%$ de la población fue correctamente clasificada- con una mínima mejora a 0,655 con el agregado del ITBPA. Mientras que en las mujeres, el PF mostró un poder de discriminación de 0,605, que se incrementó significativamente a 0,658 con el agregado del ITBPA, lo que implica sumar casi un $5 \%$ adicional de mujeres correctamente clasificadas.

Es de remarcar que en este trabajo el PF se desempeñó menos eficientemente que en otras cohortes recientemente publicadas $^{3,4}$; lo que remarca la importancia de conocer el desempeño de una herramienta diagnóstica en el medio donde uno desarrolla su práctica.

\section{Conclusiones del comentador}

Sumado a los factores de riesgo tradicionales, el ITBPA mejora la predicción del riesgo CV en mujeres de mediana edad, especialmente en las de bajo y moderado riesgo de acuerdo al PF. Por último y para llevar al consultorio adjuntamos la tabla 2 en la que se resalta en negritas los cambios de categoría luego del ajuste del resultado del puntaje de Framingham con el ITBPA.

Tabla 2: regla práctica para ajustar los resultados del puntaje de Framingham de acuerdo a los resultados del índice tobillo-brazo de presión arterial (ITBPA) medida por ecografía doppler.

\begin{tabular}{|c|c|c|c|c|c|}
\hline \multirow{2}{*}{\multicolumn{2}{|c|}{$\begin{array}{l}\text { Estimación inicial del riesgo } \\
\text { cardiovascular de acuerdo } \\
\text { al puntaje de Framingham }\end{array}$}} & \multicolumn{4}{|c|}{ Estimación luego de ajustar por ITBPA } \\
\hline & & 0 , & 0,91 a 1,1 & 1,11 a 1,4 & Mas a 1,4 \\
\hline \multirow[t]{3}{*}{ Hombres } & Alto & \multicolumn{2}{|c|}{ No cambia } & \multicolumn{2}{|c|}{ Desciende a riesgo intermedio $(14 \%)$} \\
\hline & Intermedio & \multicolumn{3}{|c|}{ No cambia } & Desciende a bajo riesgo \\
\hline & Bajo & & \multicolumn{2}{|c|}{ No cambia } & $(8 \%)$ \\
\hline \multirow{2}{*}{ Mujeres } & Intermedio & \multirow[t]{2}{*}{ Ascienden } & \multirow{2}{*}{\multicolumn{3}{|c|}{ No cambia }} \\
\hline & Bajo & & & & \\
\hline
\end{tabular}

Ignacio Bluro [ Servicio de Cardiología del Hospital Italiano de Buenos Aires. ignacio.bluro@ hospitalitaliano.org.ar ]

Bluro I. El índice tobillo-brazo puede mejorar la predicción de eventos cardiovasculares. Evid. Actual. Práct. Ambul; 12(2): 56-57, Abr-Jun 2009. Comentado de: Ankle Brachial Index Collaboration. Ankle Brachial Index Combined With Framingham Risk Score to Predict Cardiovascular Events and Mortality. JAMA 2008; 300: 197-208. PMID 18612117. Disponible en URL: http://jama.ama-assn.org/cgi/content/abstract/300/2/197

\section{Referencia}

1. Steg P y col. One year cardiovascular event rates in outpatient with atherothrombosis. JAMA 2007; 297: 1197-1206

2. Beckman J y col. on Screening for Peripheral Arterial Disease: More Harm Than Benefit? The United States Preventive Services Task Force Recommendation Statement. Circulation. 2006;114:861-866.)

3. Simmons R y col. Evaluation of the Framingham risk score in the European Prospective Investigation of Cancer-Norfolk cohort: does adding glycated hemoglobin improve the prediction of coronary heart disease events? Arch Intern Med. 2008 Jun 9;168(11):1209-16.

4. Wannamethee S y col. Metabolic syndrome vs Framingham Risk Score for prediction of coronary heart disease, stroke, and type 2 diabetes mellitus. Arch Intern Med. 2005 Dec 12-26; 165(22):2644-50. 